\title{
Drug Therapy In Rheumatoid Arthritis
}

\author{
MY Mok*, MBBS, MRCP(UK) \\ C S Lau, MD, MRCP(UK), FHKAM (Medicine)
}

\section{Summary}

Rheumatoid arthritis is such a debilitating illness that much effort has been made to find the ideal therapeutic regimen that has a high efficacy but low toxicity rate. Although such regimen has not been found yet, judicious use of what are currently available can improve the outcome of many of our patients. (HK Pract 1996; 18: 353-358)

\section{摘要}

類風濕性關節炎使患者虚弱不堪, 故需要尋求一個有功效和少妻性的理想治獠方案。至今這理想邉未能實現, 但明斷地 使用現今的藥物都可紓緩病者的不適。

\section{Introduction}

Rheumatoid arthritis (RA) is a chronic inflammatory and potentially deforming polyarthropathy. In addition to functional disability, extraarticular manifestations, though less commonly seen in Chinese patients, ${ }^{1}$ are associated with increased morbidity and mortality. A good drug therapy regimen should aim to relieve symptoms, restore functional ability and be devoid of side effects. This, together with help from other disciplines such as physiotherapy, occupational therapy and surgery, will lead to an improvement in the patient's quality of life.

A definite diagnosis is needed for any treatment regimen to be effective. The American Rheumatism Association has a revised version of criteria for the classification of RA (Table 1 ). ${ }^{9}$ Typically, there is symmetrical polyarthritis which waxes and wanes. Patients complain of early morning stiffness, swelling, warmth and redness of the affected joints. Any joint can be affected but the proximal interphalangeal joints, metacarpophalangeal joints, metartarsophalangeal joints, knees, ankles, elbows and shoulders are commonly involved. Rheumatoid factor (RF) is only present in about $80 \%$ of patients. Radiologically, there may be narrowing of joint space, periarticular osteoporosis and bony erosion. However, these findings may be absent in early disease.
Table 1: The American Rheumatism Association 1987 revised criteria for the classification of rheumatoid arthritis

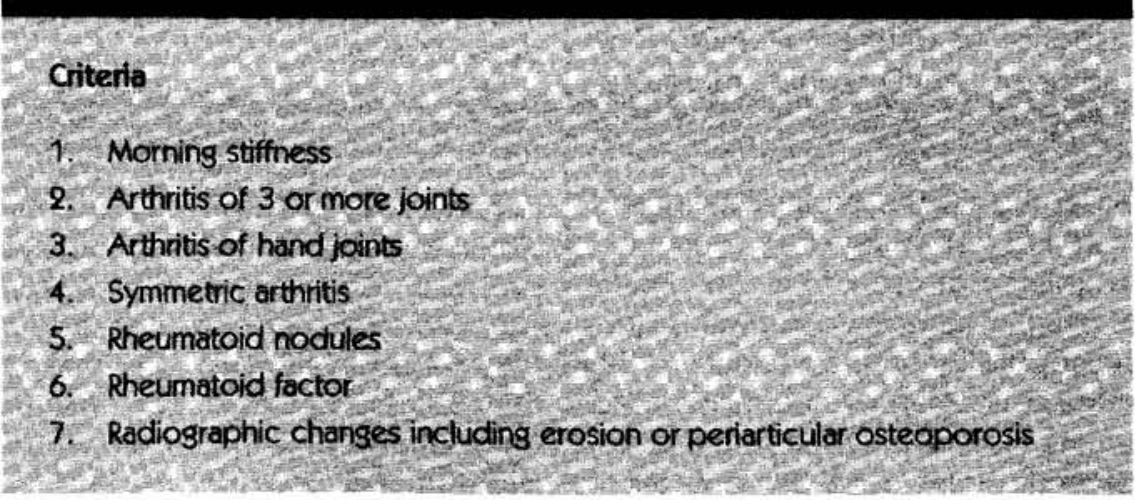

- Address for correspondence: $\quad$ DrMY Mok, Medical Officer, Department of Medicine, The University of Hong Kong, Queen Mary Hospital, Hong Kong. 


\section{CURRENT THERAPEUTICS}

\section{Drug treatment of rheumatoid arthritis}

Non-steroidal anti-inflammatory drugs (NSAIDS) are widely prescribed to alleviate the symptoms of RA. However, they do not alter the course of disease. Rather, there is a group of slow acting agents that is now recognised to alter the progression of the disease. They have individual heterogenous actions which include antibacterial, antimetabolic and immunomodulatory effects. But their exact mechanism of actions as a disease modifying agent is not known. They are collectively called disease modifying anti-rheumatic drugs (DMARDs), and they differ from NSAIDs by their slow onset of action, ability to alter the course of the disease and their relatively higher toxicityrate. Drugs such as non-NSAID analgesics and tricyclic antidepressants also have a role in symptom control although, as with NSAIDs, they have no disease modifying effects.

\section{Choice of NSAIDs}

NSAIDs act by inhibiting cyclooxygenase ( $\mathrm{CO}$ ), an enzyme that catalyzes the synthesis of proinflammatory prostaglandins from arachidonic acid. This enzyme is inhibited to a varying extent by each individual agent. However, there is no correlation between the degree of $\mathrm{CO}$ inhibition and clinical efficacy. Also, there is an individual patient response to different NSAIDs. The choice of NSAID would, therefore, only matter in terms of the frequency of the oral preparation that is soverned by the bioavailability and half life of the drug and the avoidance of use of certain classes of drus in patient groups with higher susceptibility to their toxicity. The selection of NSAID should be based on the clinician's experience and the patient's preference. ${ }^{3}$ The dosages of commonly used NSAIDs are illustrated in Table 2.

\section{Table 2: Commonly used non-steroidal anti-inflammatory agents}

NSAID

Naphthylkanones Nabumetone (Relifex)

Carboxylic acids Acetylsalicylic acid (Aspirin)

Propionic acids lbuprofen (Brufen) Naproxen (Naprosyn) Ketoprofen (Oruvail)

Acetic-acid derivatives Indomethacin (Indocid) Sulindac (Clinoril) Diclofenac (Voltaren)

fenamates Mefenamic acid (Ponstan)

Oxicams Piroxicam (Feldene) Tenoxicam (Ticotil) usual dosage

$500 \mathrm{mg}$ bid

$2-6 \mathrm{gm} /$ day in 4 divided doses

$400-800 \mathrm{mg}$ tid 250-500 mg bid $100-200 \mathrm{mg}$ bid

25-50 mg tid or qid $200 \mathrm{mg}$ bid 50-75 mg bid

$250 \mathrm{mg}$ qid

10-20 mg bid $20 \mathrm{mg}$ ad
Gastrointestinal disturbance with nausea and epigastric discomfort is the commonest side effect. NSAID induced peptic ulcer may also occur with regular long term treatment especially in the elderly ( $>60$ years). The incidence may be reduced by taking misoprostol, a prostaglandin analogue, concomitantly. ${ }^{4} \quad \mathrm{H}_{2}$ antagonists have not been shown to prevent NSAID related gastric ulcers but they can help to heal these ulcers more rapidly once they occur. ${ }^{4}$ The role of co-administration of antacid is not yet defined. Indeed, antacids may mask dyspeptic symptoms and delay diagnosis.

More potent inhibitors of $\mathrm{CO}$ are associated with higher risks of anaphylaxis in atopic patients. Nonacetylated salicylates such as propionic acids e.g. ibuprofen, can be considered in these patients as they are relatively weak inhibitors of CO. Because of the effect of NSAIDS on decreasing synthesis of prostaglandin which plays a protective function on the kidney, the resulting vasoconstriction may lead to renal ischaemia. The risk of renal impairment increases in patients with other risk factors like uncompensated congestive heart failure, liver cirrhosis and ascites. ${ }^{4}$

Combination use of NSAIDs should be avoided as this greatly increases toxicity without much increase in efficacy.

\section{When to use DMARDs?}

The classical management of RA takes the form of a therapeutic pyramid starting with NSAIDs as the initial therapy followed by DMARDs with increasing efficacy and toxicity as the pyramid ascends. There is, however, a recent trend of using DMARDs early in the course of RA and using more aggressive agents in rapidly progressive disease. This hit hard and early regimen aims to prevent development of bony erosion and hence deformity, which has been shown by radiological study to occur during the early course of disease. $^{5}$ 


\section{CURRENT THERAPEUTICS}

Because of the higher rate of toxicity of DMARDs, they should be used in patients who are likely to benefit from them so that a low risk/ benefit ratio can be achieved. The overall drop out rate is about $40 \%$ at 2 years, mainly due to lack of efficacy or side effects. Therefore, these drugs should only be used in patients who are likely to benefit from these agents. ${ }^{\circ}$ They include those who possess markers of active disease or poor prognostic factors (Table 3 ).

As DMARDs are slow acting, impatient switching to another agent should be avoided. Most DMARDs take at least three months to become effective. In cases of resistant disease that have failed to respond to high efficacy DMARDs, combination therapy can be tried although there is so far no demonstrable increase in efficacy over single agents. ${ }^{7}$ These patients should better be referred to rheumatologists for management and monitoring of possible additive side effects.

\section{Choice of DMARDs}

There is no evidence to suggest any DMARD is superior to others in terms of efficacy. Therefore, as with NSAIDs, the choice of DMARDs is often based on the physician's experience of the drug tailored by individual circumstances such as disease presentation and comorbidity. ${ }^{8} \quad$ (Table 4 ) summarizes the side effects of commonly used DMARDs.

\section{Hydroxychloroquine(HCQ)}

This antimalarial agent has modest efficacy as a disease modifying agent in RA. It is usually used in mild or oligoarticular disease. The maintanence dosage is $200 \mathrm{mg}$ per day. Prolonged use may give rise to a 'bull's eye' retinopathy and referral to ophthalmologist for baseline assessment and regular check up is recommended.

\section{Sulphasalazine}

This sulphonamide agent is also commonly used in early RA. The starting dose is $500 \mathrm{mg}$ per day to be gradually stepped up every week by $500 \mathrm{mg}$ until a daily dose of 2 to $2.5 \mathrm{gram}$ is reached.

\section{Auranofin}

This oral gold preparation is less popularly used nowadays. This is mainly due to the slow onset of action of about 6 to 9 months and the relatively high rate of side effects including nausea, abdominal pain and diarrhoea although thrombocytopenia and proteinuria are less commonly encountered compared to injectable gold. Loose bowel motion may improve with the use of bulk forming agents. A total dose of $6 \mathrm{mg}$ is usually used per day.

\section{Intramuscular myocrisin}

This parenteral form of gold salts gives faster onset of action than its oral counterpart. A test dose of $20 \mathrm{mg}$ to exclude anaphylaxis is usually given followed by $40 \mathrm{mg}$ per week until a total accumulated dose of $500 \mathrm{mg}$ is reached. The dose interval can then be extended to fortnightly. Further spacing out to 4 weekly can be made when a total dose of $1 \mathrm{gm}$ is reached. Exacerbation of RA can be managed by re-induction therapy by increasing the dosage interval to weekly administration.

\section{Penicillamine}

This medication is less commonly used nowadays because of proportionately higher incidence of side effects. The usual dosage is $750 \mathrm{mg}$ per day.

\section{Methotrexate (MTX)}

MTX is an antifolate agent. However, its exact mechanism on RA is not known. It is increasingly used nowadays because of its high efficacy and short onset of action. Radiological studies have suggested true regression of RA following MTX treatment. $^{\circ}$ MTX takes about 6 weeks for its effect to be demonstrated clinically.

\section{Table 3: Indications for disease modifying anti-rheumatic drugs}

Indication

1. Persistently 6 or more tender or swollen joints

2. Duration of morning stiffness over 45 minutes

3. High serum levels of acute phase reactants (CRP $>1 \mathrm{mg} / \mathrm{dl}$ and ESR $>30 \mathrm{~mm} / \mathrm{hr}$ )

4. High titre of RF at presentation

5. Early radiological demonstration of bony erosion

6. Presence of extra-articular manifestations. 
Table 4: Side effects of disease modifying anti-rheumatic drugs

DMARDS

Hydroxychloroquine

Sulphasalazine

Oral and parenteral gold

Penicillamine

Methotrexate

Azathioprine

Cyclophosphamide

\section{Side effects}

Retinopathy, myopathy

Nausea, vomiting, haemolytic anaemia, bone marrow suppression, hepatotoxicity, azospermia

Nausea and diarrhoea, skin rash, oral ulcer, bone marrow suppression, hepatotoxicity, nephrotic syndrome

Skin rash, oral ulcer, taste disturbance, drug induced lupus, myasthenia gravis, Goodpasture's syndrome, nephrotic syndrome

Bone marrow suppression, hepatotoxicity, pneumonitis

Bone marrow suppression, hepatotoxicity, higher incidence of malignancy, infection

Bone marrow suppression,

haemorrhagic cystitis,

higher incidence of malignancy,infection
Regular monitoring

Regular ophthaimology check up

Full blood count;iver function test

Full blood count; liver function test; urine for protein

Full blood count; urine for proteinuria

Pretreatment hepatitis $B$ and $C$ status check is desirable; full blood count; liver function test

Full blood count, liver function test

Full blood count

Concomitant use with allopurinol

before starting the drug. Although the presence of any of these chronic viral hepatitis is not an absolute contraindication to the use of MTX, a baseline liver biopsy may need to be considered and subsequent judicious monitoring of the liver function is mandatory. Patients should avoid alcohol use during MTX treatment. MTX should be should be avoided as the toxicity would be enhanced.

level and re-introduced at a lowe dose after the enzyme level has returned to normal.

MTX is usually started at $2.5 \mathrm{mg}$ per week and stepped up by $2.5 \mathrm{mg}$ per week till a dosage of 7.5 to $10 \mathrm{mg}$ is reached. A higher dose can be used but folic acid supplement, administered on days between MTX treatment, is recommended. ${ }^{11}$

withheld if there is an elevation of

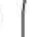

\section{Cyclophosphamide}

This is an alkylating agent and, like azathioprine, is only used in resistant cases. Patients should take plenty of fluid to reduce the incidence of haemorrhagic cystitis.

\section{Cyclosporin}

\section{Azathioprine}

This is a cytotoxic agent which is usually used later in the course of disease, mainly because of its higher toxicity rate. It is usually given at $50 \mathrm{mg}$ or $100 \mathrm{mg}$ per day.
This is an immunomodulator which is also used late in the disease effects, narrow therapeutic margin and high cost. Cyclosporin use should be limited to those familiar with this medication. because of its unfavourable side 


\section{Role of steroid in rheumatoid arthritis}

The exact mechanism of steroid as an anti-inflammatory agent in rheumatic diseases is not known. Opinions vary on its use in RA. In general, long term use should be discouraged. Some consider a low dose of oral steroid can help in the suppression of symptoms of inflammation and even disease progression. However, others would only consider low dose oral steroid or intramuscular depot steroid as bridging therapy for DMARDs before they take their effect. The use of long term steroid agents is mainly limited by their long list of side effects. ${ }^{12}$

Intra-articular steroid is commonly given locally to achieve a quicker symptomatic relief. The triamcinolone hexacetonide preparation has an advantage over hydrocortisone or acetonide preparation because of its low solubility that gives longer lasting local pain relief. Repeated steroid injections $(>3)$ into the same joint are discouraged due to their potential adverse effects on the cartilage.

\section{How to assess the response to medical therapy?}

The American College of Rheumatology and the European League Against Rheumatism have set criteria for the assessment of activity and outcome of RA which can be used as guidelines in the monitoring of response of disease to drug therapy (Table 5). ${ }^{13}$ Any single parameter would not be good enough indicator of response of disease and an overall assessment is needed. In clinical practise, a positive response should include improvement in both the patient's symptomatology and function as well as laboratory parameters of disease activity.
Table 5: European League Against Rheumatism recommended criteria for assessment of RA activity and outcome

\section{Criteria}

1. number of swollen joints

2. number of tender joints

3. patient's global assessment score

4. clinician's global assessment score

5. acute phase response

6. functional assessment

7. radiological assessment

\section{standardization}

with or without joint effusion

pain on pressure or movement of joint

a five point score, graded: 1 asymptomatic, 2-mild, 3 moderate, 4-severe, 5-very severe

same as above

ESR $(\mathrm{mm} / \mathrm{hr}), \mathrm{CRP}(\mathrm{g} / \mathrm{l})$

self administered questionnaries e.g. Health Assessment Questionnaire

$X$-ray damage measured by the Larsen or the Sharp method

\section{References}

1. Cohen MG, Li EK, Ng PY and Chan KL: Extraarticular manifestations are uncommon in southern Chinese with rheumatoid arthritis. Br J Rheumatol 1993; 32: 209-211.

2. Arnett $F C, E$ dworthy $S M$, Bloch DA, McShane DJ, Fries Jf, CooperNS, Healey LA, Kaplan SR, Liang MH, Luthra HS, Medsger TA Jr, Mitchell DM, Neustadt DH, Pinals RS, Schaller JG, Sharp JT, Wilder RL and Hunder GG: The American Rheumatism Association 1987 revised criteria for the classification of rheumatoid arthritis. Arthritis Rheum 1988; 31, 315-324.

3. Keys J, Beardon P, Lau CS, Lang CC and MCDevitt DG. General practitioner use of non-steroidal anti-inflammatory drugs in Tayside and Fife regions. I ROY SOC Med 1992; 85: 442-445.

4. Lichtenstein DR, Synagal $S$ and Wolfe $M M$. Nonsteroidal anti-inflammatory drugs and the gastrointestinal tract. Arthritis Rheum 1995; 38: $5-18$

5. Van der Heide A, Remme CA, Hofman DM, Jacobs JWG and Bijlsma JWJ. Prediction of progression of radiologic damage in newly diagnosed rheumatoid arthritis. Arthritis Rheum 1995; 38: 1466-1474

6. Capell H, Porter D, Madhok R, Hunter $J$. Second line (Disease Modifying) Treatment in Rheumatoid Arthritis: Which Drug for Which Patient? Ann Rheum Dis 1993; 52 : 423-428.

7. Willkens RF, Sharp JT, Stablein D, Marks C and Wortmann R. Comparison of azathioprine, methotrexate, and the combination of the two in the treatment of rheumatoid arthritis. Arthritis Rheum 1995; 38: 1799-1806.

8. Conaghan $P G$ and Brooks $P$. Disease-modifying antirheumatic drugs, including methotrexate, gold, antimalarials and D-penicillamine. Curr Opin Rheumatol 1995; 7: 167-173.

9. Alarcon GS, Lopez-Mendez A, Walter J, Boerbooms AMT, Russell AS, Furst DE, Rau R, Drosos AA and Bartolucci AA. Radiographic evidence of disease progression in methotrexate treated and nonmethotrexate disease modifying antirheumatic drug treated rheumatoid arthritis patients: a meta-analysis. J Rheumatol 1992; 19: 1868-1873.

10. Kremer JM, Alarcon GS, Lightfoot RW, Willkens RF, Furst DE, Williams HJ, Dent PB and Weinblatt ME. Methotrexate for rheumatoid ar thritis. Arthritis Rheum 1994; 37: 316-328.

11. Dijkmans BAC. Folate supplementation and methotrexate. Br J Rheumatol 1995; 34:11721174.

12. Weisman $\mathrm{MH}$. Corticosteroids in the treatment of rheumatologic diseases. Curr Opin Rheumatol 1995, 7: 183-190.

13. Van Riel PLCM. Provisional guidelines for measuring disease activity in RA clinical trials. Br J Rheumatol 1992; 10:521-525. 